\title{
The role of excluded volume and electrostatics from coarse-grain modeling of the interaction of gemini surfactants with like-charged membranes
}

\author{
Sandra C.C. Nunes, J.A.S. Almeida, R.S. Dias \& A.A.C.C. Pais
}

To cite this article: Sandra C.C. Nunes, J.A.S. Almeida, R.S. Dias \& A.A.C.C. Pais (2013) The role of excluded volume and electrostatics from coarse-grain modeling of the interaction of gemini surfactants with like-charged membranes, Molecular Physics, 111:1, 123-134, DOI: 10.1080/00268976.2012.707339

To link to this article: https://doi.org/10.1080/00268976.2012.707339

Accepted author version posted online: 02

Jul 2012.

Published online: 30 Jul 2012.

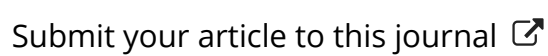

山 Article views: 84

Citing articles: 1 View citing articles ¿ð 


\title{
RESEARCH ARTICLE
}

\section{The role of excluded volume and electrostatics from coarse-grain modeling of the interaction of gemini surfactants with like-charged membranes}

\author{
Sandra C.C. Nunes*, J.A.S. Almeida, R.S. Dias and A.A.C.C. Pais \\ Chemistry Department, University of Coimbra, Rua Larga 3004-535, Coimbra, Portugal
}

(Received 10 May 2012; final version received 21 June 2012)

\begin{abstract}
Cationic systems composed of lipids and/or surfactants are of paramount importance in a variety of applications. Within these, gemini have attracted particular attention, mainly due to their improved aggregation properties and to the possibility of tuning offered by the presence of a spacer. In this work, a Monte Carlo simulation study with a coarse-grained model was employed to assess the interaction of cationic gemini surfactants with a like-charged model membrane. Separating the contribution of the excluded volume and that of the electrostatic effects in the organization of gemini-lipid membranes was the first goal of this work and the role of these factors was assessed varying the concentration, the spacer length and the headgroup charge of gemini surfactants. The results provide a new insight on the organization of lipid headgroups in the vicinity of gemini surfactants. It was found that the surfactant-lipid interaction is strongly affected by the surfactant spacer length, being controlled by an overall balance between excluded volume and surfactant-lipid and surfactant-surfactant electrostatic effects. It is also seen that the out-of-plane motion of the spacer has a significant effect upon membrane organization and counterion condensation. Good agreement was found with results previously obtained from atomistic simulation.
\end{abstract}

Keywords: dicationic gemini surfactant; model membrane; coarse-grained; electrostatics; excluded volume

\section{Introduction}

The current work relies on a coarse-grained model of the structural properties of positively charged membrane systems modulated by dicationic gemini surfactants.

The interest in gemini surfactants is widely recognized in several areas ranging from gene therapy [1-6], drug delivery $[2,7]$, and antibacterial treatments $[8,9]$ to industrial applications [10-14]. The amount of information and work developed in the last few years has also given rise to a vast number of review papers on the topic [6,15-18]. This class of molecules has been the focus of various studies due to their improved aggregation properties and better solubilizing and dispersing ability compared to conventional surfactants [15]. Moreover, gemini surfactants can be synthesized in a large variety of structures, in which two single tail surfactants are connected by a spacer group of varying nature and length $[15,19,20]$, meaning that the properties of gemini surfactants can be modelled to fit a specific application [21,22].

Computer simulations have been widely used to complement and rationalize experimental results. Approximate descriptions of the systems in coarsegrained models, with significant less detail than their atomistic counterpart, have been recognized as suitable to characterize the behaviour of very complex systems. Examples of application range from models for nucleic acids [23,24], membranes [25,26], or proteins [27-29]. Previous work has also focused on more general aspects of coarse-grained approaches [30,31]. Although at the microscopic level, the interactions between the components of a system can be very complex, the essential aspects can often be characterized by a relatively small set of parameters. In this context, this simplified coarse-grained study is used not only as a means to reduce the computer simulation time, but also to allow a better control over system features and to assess their impact on the overall behaviour.

\section{Model and method}

A simple model was adopted to describe the interaction between gemini surfactants and lipids in a model membrane. All charged species are explicitly included. Focus is given to the surface of the membrane and, thus only the headgroups and spacers are included. As such, the gemini surfactant, represented by a springbead chain, is described as a sequence of positively

*Corresponding author. Email: snunes@qui.uc.pt 
charged hard spheres, in the case of a short spacer, or a sequence of positive and neutral beads connected with harmonic bonds, when longer spacers are present. In the latter cases, the chain flexibility is additionally regulated by angular force terms. The charged membrane surface is composed of a hard-planar wall, with embedded positively charged hard-spheres (lipids), which are laterally mobile. This model assumes that there will be no substantial changes in the curvature of the membrane, at least up to the surfactant concentration that was considered in this work. For simplicity, the model also assumes that the headgroups of the gemini surfactants are incorporated at the same level as the lipid headgroups, which does not necessarily occur for the gemini surfactants with the shortest spacers [32-34]. Monovalent simple ions, also treated as charged hard spheres, were also included in order to obtain an electroneutral system. The solvent enters the system only through its relative permittivity.

A rectangular box with box lengths $L_{x}=L_{y}=200 \AA$, and $L_{z}=800 \AA$ was considered. The system is periodic in the $x y$ plane, while hard walls were placed at $z=150 \AA$. The number and the size of the lipid beads was chosen in order to reproduce the lipid-lipid typical distance obtained by a Molecular Dynamics study [32]. Accordingly, a hard-sphere (hs) radius of $3.3 \AA$ was used for the gemini surfactant beads and lipid headgroups, and a hs radius of $2.2 \AA$ was considered for the monovalent counterions [35]. The model lipid membrane was composed of 554 lipid headgroups. According to the imposed number of lipid headgroups and membrane area, the surface charge density of the membrane is $c a .0 .22 \mathrm{C} \mathrm{m}^{-2}$ and the area per lipid is $c a .0 .72 \mathrm{~nm}^{2}$. Both values are compatible with those reported in the literature for fluid phases of lipid membranes $[32,36,37]$. The percentage of gemini surfactants was varied throughout the work. For each added surfactant, two lipid headgroups were removed to keep the overall charge density of the membrane constant. The number of anionic monovalent counterions was thus kept constant and equal to 554. A temperature $T=298.15 \mathrm{~K}$ and a relative permittivity $\varepsilon_{\mathrm{r}}=78.4$ were considered throughout.

All interactions were taken as pairwise additive. The total potential energy, $U$, of the system is expressed as a sum of four contributions: the nonbonded potential energy, $U_{\text {non-bond}}$, the bonded potential energy, $U_{\text {bond }}$, the angular potential energy, $U_{\text {ang, }}$, and the confining external potential energy, $U_{\text {ext }}$. The non-bonded potential energy is given by

$$
U_{\text {non-bond }}=\sum_{i<j} u_{i, j}\left(r_{i, j}\right),
$$

where the summation extends over surfactant beads, lipid beads and counterions, with $u_{i, j}$ representing the electrostatic potential plus a hard-sphere repulsion according to

$$
u_{i, j}\left(r_{i, j}\right)= \begin{cases}\infty, & r_{i, j}<R_{i}+R_{j}, \\ \frac{Z_{i} Z_{j} e^{2}}{4 \pi \epsilon_{0} \epsilon_{\mathrm{r}}} \frac{1}{r_{i, j}}, & r_{i, j} \geq R_{i}+R_{j},\end{cases}
$$

where $Z_{i}$ is the valence of the particle $i, R_{i}$ is the radius of particle $i, r_{i, j}$ is the distance between particles $i$ and $j$, $e$ is the elementary charge, $\varepsilon_{\mathrm{o}}$ is the permittivity of vacuum, and $\varepsilon_{\mathrm{r}}$ the relative permittivity of the solvent. Hard-sphere repulsion was not imposed for connected consecutive beads. Surfactant beads are connected by harmonic bonds, and the bond potential energy of the surfactant is given by

$$
U_{\mathrm{bond}}=\sum_{i=1}^{N_{\mathrm{bead}}-1} \frac{k_{\mathrm{bond}}}{2}\left(r_{i, i+1}-r_{0}\right)^{2}
$$

where $N_{\text {bead }}$ is the number of beads, $r_{i, i+1}$ is the distance between two connected beads, with the reference separation $r_{0}=3.5 \AA$, in the case of the gemini surfactant with the shortest spacer, and $r_{0}=5.0 \AA$ in the remaining cases, and the force constant $k_{\text {bond }}=0.4$ $\mathrm{Nm}^{-1}$. The angular potential energy, $U_{\mathrm{ang}}$, is given by

$$
U_{\text {ang }}=\sum_{i=1}^{N_{\text {bead }}-1} \frac{k_{\text {ang }}}{2}\left(\alpha_{i, i+1}-\alpha_{0}\right)^{2},
$$

where $\alpha_{i}$ is the angle formed by the vectors $r_{i+1}-r_{i}$ and $r_{i-1}-r_{i}$ with the equilibrium angle $\alpha_{0}=180^{\circ}$ and the force constant $k_{\text {ang }}=3.44 \times 10^{-24} \mathrm{~J} \mathrm{deg}^{-2}$. Finally, the confining external potential energy, $U_{\text {ext }}$, is given by

$$
U_{\mathrm{ext}}=\sum_{i} u_{\mathrm{ext}}\left(z_{i}\right)
$$

where the summation extends only over counterions with

$$
u_{\text {ext }}\left(z_{i}\right)= \begin{cases}\infty, & \left|z_{i}\right|>z_{\text {wall }}, \\ 0, & \left|z_{i}\right|<z_{\text {wall }} .\end{cases}
$$

All Monte Carlo simulations were performed with the MOLSIM [38] simulation package in the canonical ensemble, employing the Metropolis algorithm [39]. Two types of Monte Carlo trial moves were employed for the surfactant: single bead moves and translation of the entire chain, both restricted to the $x y$ plane, although in some cases the beads representing the surfactant spacer were allowed to move along the $z$-direction (labelled as $z_{\text {free }}$ ). In this case only single bead moves were allowed. The counterions and lipids were subjected to translational moves, with those of the latter restricted to the $x y$ plane. Each simulation 
Table 1. Summary of the systems under study, including variation in the gemini $(G)$ content and spacer length. Neutral beads in the spacer are denoted by (o) and charged headgroups by $(+)$. $\mathrm{N}_{\mathrm{G}}$ corresponds to the number of surfactant molecules introduced in the membrane.

\begin{tabular}{lccr}
\hline System & Scheme & $\mathrm{N}_{\mathrm{G}}$ & $\% \mathrm{G}$ \\
\hline I & $1(++)$ & 1 & 0.4 \\
II & $30(++)$ & 30 & 10.8 \\
III & $50(++)$ & 50 & 18.1 \\
IV & $(+\mathrm{o}+)$ & 30 & 10.8 \\
V & $(+$ oo +$)$ & 30 & 10.8 \\
VI & $(+$ ooo +$)$ & 30 & 10.8 \\
VII & $(+$ ooo +$)-z_{\text {free }}$ & 30 & 10.8 \\
\hline
\end{tabular}

included an equilibration of at least $5 \times 10^{5}$ trial moves per particle followed by a production run of at least $5 \times 10^{5}$ trial moves per particle.

The arrangement of the lipid headgroups, both in the absence and presence of the gemini surfactants, was analysed resorting mainly to two-dimensional radial distribution functions (2D-rdf) and to positioning density maps. The distribution of the counterions relatively to the lipids and surfactant headgroups was evaluated using $3 \mathrm{D}$-rdfs.

\section{Results and discussion}

The effect of the cationic gemini surfactants upon a positively charged lipid membrane was analysed considering the systems summarized in Table 1. Systems I to III were used to assess the effect of gemini concentration in the organization of the lipid membrane. In systems IV to VII the effect of the surfactant spacer length was inspected for the system of intermediate concentration. In the latter set of systems, spacer motion along the $z$-axis was also tested. We recall that the total number of charged headgroups was kept constant, when the surfactant concentration increases, by replacing lipid headgroups with the same number of surfactant headgroups.

Rdf analyses and density contour profiles allowed one to establish the positioning of the components relative to each other as a function of the spacer length, concentration and headgroup charge of the surfactant.

\subsection{Effect of gemini concentration}

Figure 1 displays the 2D-rdf for the lipid headgroups upon the increase in concentration of gemini surfactants (corresponding to systems I-III). The lipid-lipid rdf has a main peak at $c a$. $7 \AA$, which corresponds

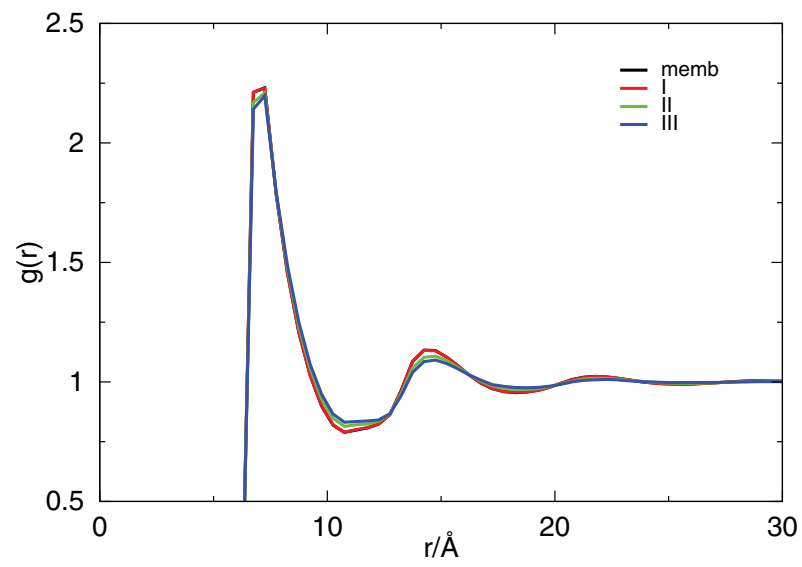

Figure 1. 2D-rdf for lipid-lipid headgroups for systems containing an increasing gemini surfactant content. The 2Drdf of the lipid-lipid particles without gemini, as control system, is also included (memb).

approximately to the sum of the lipid headgroup radii, and two smaller ones at around 14 and $22 \AA$ corresponding to a profile of successive concentric hardspheres. The ordering is not perturbed upon the increase of the gemini concentration, showing only a slight decrease in the probability of the first peak and of the long-range order, with the near disappearance of the third peak for the most concentrated system. It is noteworthy that the typical distance between the lipid headgroups, in the absence of surfactant, is maintained with the incorporation of gemini surfactants, even for concentrations of the latter around $20 \%$.

In Table 2 are indicated the average values for the root-mean-square end-to-end distance of the surfactant $\left(\left\langle R_{\mathrm{ee}}^{2}\right\rangle^{1 / 2}\right)$, i.e. the distance between the two charged headgroups, for systems I-III. It can be seen that the $\left\langle R_{\mathrm{ee}}^{2}\right\rangle^{1 / 2}$ values increase as the gemini content increases. This trend is associated with the decrease of the number of lipid molecules as the gemini content increases. Lipids are replaced by gemini in which the headgroups are closer than vicinal lipids, due to the fact that the beads are connected and have the possibility to overlap, thus promoting a slight decrease in the hs density. This, in turn, allows a larger average extension between the headgroups of the surfactant.

\subsection{Effect of gemini spacer length}

The 2D-rdf curves reflecting the influence of the gemini spacer length on the membrane organization are represented in Figure 2. In panel (a) it is seen that the surfactant spacer length has a small influence on the profile of the distribution of the lipid headgroups 
Table 2. Gemini root-mean-square endto-end distances, $\left\langle R_{\mathrm{ee}}^{2}\right\rangle^{1 / 2}$, for systems I to III. The uncertainty is given as the standard error of the mean.

\begin{tabular}{ll}
\hline system & $\left\langle R_{\mathrm{ee}}^{2}\right\rangle^{1 / 2} / \AA$ \\
\hline I & $3.746 \pm 0.004$ \\
II & $3.7748 \pm 0.0006$ \\
III & $3.7908 \pm 0.0007$ \\
\hline
\end{tabular}

around each other, but the intensity of the main peak is higher for surfactants with longer spacers due to the increase in the hs density. This is particularly evident when surfactants with the longest spacers are considered, VI and VII. In system VII, however, where the spacer is not-restricted to the $x y$-plane, a decrease in the intensity of the first peak can be seen. It is clear that when the spacer is allowed to move along the $z$-axis, the neutral beads representing the spacer are preferentially out of the membrane surface plane, thus promoting a smaller hs density in this system. This is suggested by the results presented in Figure 3 where the probability density of each surfactant bead along the $z$-axis is represented. In fact, the probability of finding the spacer groups in the membrane section, delimited by the green dashed lines, is very low, and the spacer is preferentially found below the membrane surface. This may be attributed to the depletion caused by the condensation of the counterions onto the surface. It should be noted that the counterions are restricted to the outer part of the membrane due to the hard wall (black dotted line). In a real lipid membrane, such directional motion of the spacer would also be expected, but in this case due to its hydrophobic nature. Regarding the profile of the counterions, a bimodal distribution is observed. The peak with the lowest position is directly related to the accumulation of the counterions due to the presence of the hard wall (black dotted line), while the other coincides with the sum of the hs radii of the gemini/membrane headgroups and that of the counterions (black dashed line). It is interesting to note that the obtained profile is very different from those commonly observed for counterion condensation in homogeneous surfaces.

The values of the two-body potential energy, $E_{i, j}$, are shown in Table 3, together with the values of $\left\langle R_{\mathrm{ee}}^{2}\right\rangle^{1 / 2}$ obtained for the different systems. The energy values show that the lipid-lipid (L-L) repulsion increases with the increase of spacer length reflecting the greater proximity of the lipid-lipid headgroups due to the higher hs density promoted by the neutral beads of the spacer. This trend is not followed by system VII
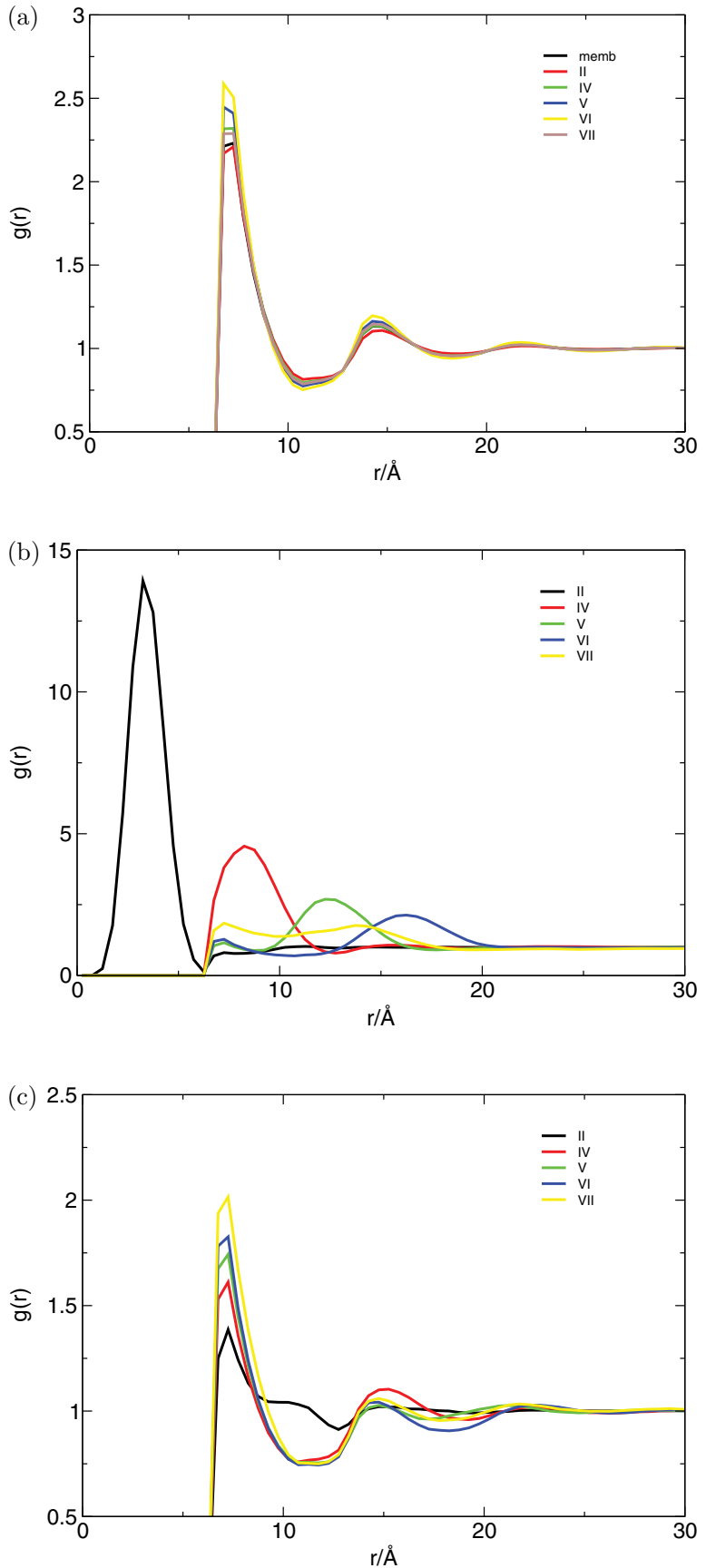

Figure 2. 2D-rdf for a system containing $10.8 \%$ of gemini surfactants, with different spacer lengths (systems II and IV to VII), inserted into a positively charged membrane: (a) lipid-lipid rdf; (b) gemini-gemini rdf; (c) gemini-lipid rdf.

according to what has been explained and addressed by the results presented in Figures 2 and 3. The $E_{i, j}$ values, are thus, in good agreement with the trends obtained with the rdf analysis.

In what concerns the gemini-gemini rdf, Figure 2 panel (b), the profiles obtained are markedly different. 


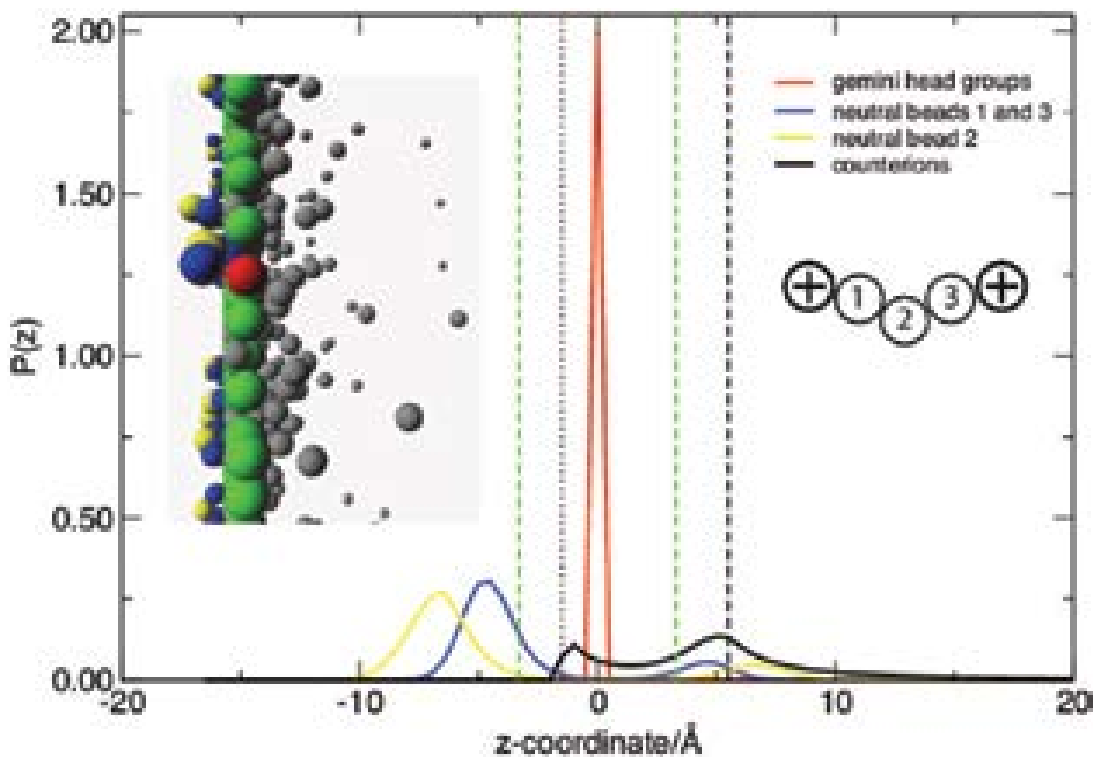

Figure 3. Probability density profiles, along the $z$-axis, for the spacer and headgroups of the gemini surfactant and the counterions in system VII. Green dashed lines define the thickness of the membrane, i.e. the space occupied by the gemini headgroups (hs radius of $3.3 \AA$ ); the black dotted line defines the position of the wall imposed to the counterions and the black dashed line corresponds to the sum of the hs radii of the gemini headgroups and that of the counterions, which coincides with the preferential positioning of the counterions (see text for details). The insert displays an illustrative snapshot of a positively charged membrane (green) containing 10.8\% of gemini surfactants. Gemini headgroups are represented in red, the blue and yellow beads represent the neutral spacer and the counterions are represented in grey.

Table 3. Parameters characterizing the systems considered for assessing the influence of surfactant spacer length. Gemini root-mean-square end-to-end distances, $\left\langle R_{\mathrm{ee}}^{2}\right\rangle^{1 / 2}$, and twobody potential energies, $E_{i, j}$ between the particles of the system: gemini headgroups $(\mathrm{G})$, lipid headgroups (L) and negative counterions (C). The uncertainty is given as the standard error of the mean.

\begin{tabular}{lccccc}
\hline & & \multicolumn{4}{c}{$E_{i, j}\left(\mathrm{~kJ} \mathrm{~mol}^{-1}\right)$} \\
\cline { 3 - 6 } System & $\left\langle R_{\mathrm{ee}}^{2}\right\rangle^{1 / 2} / \AA$ & $\mathrm{G}-\mathrm{G}$ & $\mathrm{L}-\mathrm{L}$ & $\mathrm{G}-\mathrm{L}$ & $\mathrm{G}-\mathrm{C}$ \\
\hline II & $3.7748 \pm 0.0006$ & $608 \pm 3$ & $32266 \pm 2$ & $7767 \pm 6$ & $-8063 \pm 7$ \\
IV & $8.991 \pm 0.002$ & $515 \pm 3$ & $32315 \pm 4$ & $7737 \pm 7$ & $-7979 \pm 11$ \\
V & $12.895 \pm 0.004$ & $490 \pm 5$ & $32349 \pm 8$ & $7739 \pm 12$ & $-7949 \pm 8$ \\
VI & $16.611 \pm 0.006$ & $477 \pm 2$ & $32384 \pm 5$ & $7744 \pm 6$ & $-7903 \pm 8$ \\
VII & $13.10 \pm 0.01$ & $485 \pm 2$ & $32290 \pm 3$ & $7796 \pm 5$ & $-7982 \pm 7$ \\
\hline
\end{tabular}

The position of the main peak in the curve corresponds approximately to the end-to-end average distance of the surfactant, with a dispersion that increases with the spacer length. The surfactant for which the spacer is allowed to move along the $z$-axis (VII) displays the largest dispersion, and the gemini-gemini average headgroup distance is clearly reduced if compared with the surfactant with the same spacer length, but restricted to the $x y$-plane (VI). The increase in the spacer length confers complex profiles to geminigemini rdf curves. For example, in system $\mathrm{V}$ the curve presents a first small peak at around $7.3 \AA$, and a second one, more pronounced at $c a$. $12.4 \AA$, corresponding approximately to, as mentioned, the end-toend distance $(12.895 \AA)$. Similarly, in system VI, two peaks are visible in the rdf curve, the higher one, at around $16.3 \AA$, nearly corresponds to the gemini endto-end distance for this system, $16.611 \AA$. In system VII, two broad peaks are present in accordance to the larger freedom of the spacer.

The complex profile of the gemini-gemini rdf curves can be elucidated resorting to Figure 4, where 

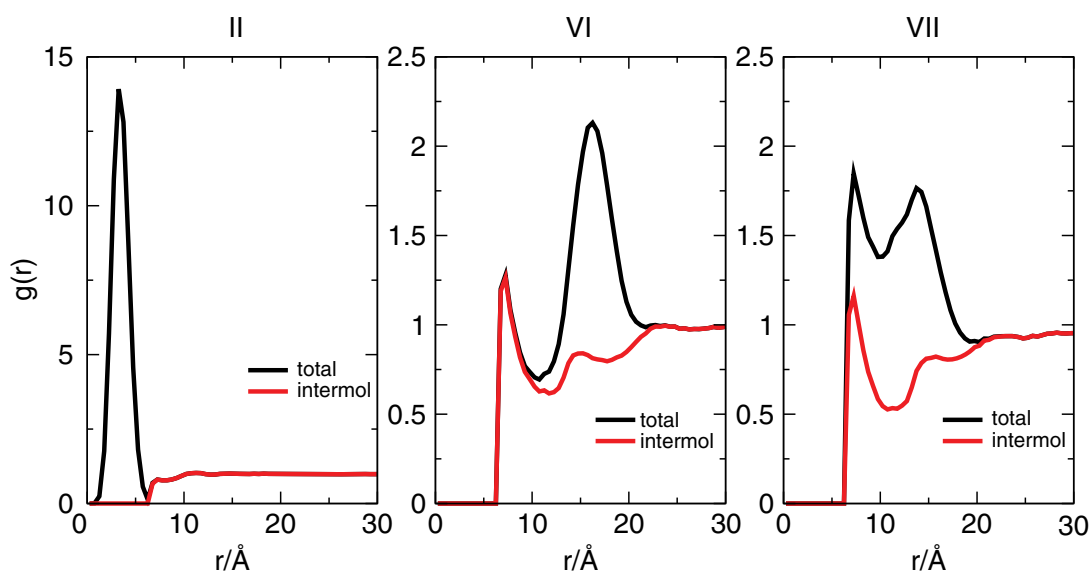

Figure 4. 2D-rdf for the gemini-gemini headgroups in systems containing $10.8 \%$ of gemini surfactants with different spacer lengths inserted into a positively charged membrane. The rdf curves represent all contributions (black curve) and only the intermolecular contributions (red curve) to the gemini-gemini rdfs.

the intermolecular contributions to the gemini-gemini interaction are discriminated. We will focus essentially on systems II, VI and VII since these cases cover the most significant differences, in what concerns the spacer length. Representative snapshots of these three systems can be visualized in Figure 5, which also serve to illustrate the typical representation of both the membrane and surfactants in this coarse-grained approach. In Figure 4, it can be seen that while in system II the peak is essentially due to the other headgroup of the same gemini molecule (intramolecular), in system VI two peaks arise: the first at $7.2 \AA$, corresponding to the distance between the different gemini molecules (intermolecular), and the second peak, at $c a$. $16.3 \AA$, corresponds to the intramolecular distance between the two headgroups of the same gemini molecule. In system VII the profile of the $\mathrm{rdf}$ is significantly different. It is clear that, comparing to system VI, the intermolecular gemini contributions are nearly the same whether the neutral linker is restricted or not to the surface. In the latter, however, there is an important contribution of intramolecular distances to the first peak too, showing that this system has a large propensity to adopt the typical lipid-lipid distance. The second peak, which is solely due to the contribution of intramolecular distances is broader and slightly shifted toward smaller distances, since the additional freedom of the spacer along the $z$-axis confers a great diversity of relative positions of the gemini headgroups and promotes smaller end-to-end distances, as compared to system VI.

The energy values reported in Table 3 for the gemini-gemini interaction, reflect the decrease of repulsion with the increase of spacer length, with the exception of system VII, where the energy increases in comparison to system VI. Note that the trend of these values follows that of the average end-to-end distance of the surfactants, indicating the prevalence of the intramolecular contribution. Although the geminigemini interaction energy increases, in system VII compared to VI, the surfactant tends to adopt a configuration that is close to that observed for the lipids in the membrane, as mentioned above (Figure 4).

In what concerns the distribution of the lipid headgroups around the surfactant, Figure 2 panel (c), the largest differences in the rdf profiles occur for the shortest spacer gemini. In this system, the curve shows a pronounced shoulder coupled to the first peak which disappears with the increase of the spacer length. It is also noteworthy that the probability of finding the lipid headgroups near the surfactant is higher for surfactants with longer spacers. The increase in the surfactant spacer length promotes a decrease of the charge density near the gemini headgroups and, consequently, the approximation of the lipid headgroups to the surfactant occurs. Additionally, when the spacer is allowed to move along the $z$-axis, system VII, the probability of finding the lipid headgroups closer to the gemini heads increases. As the spacer is more often found outside the membrane plane (Figure 3) the gemini headgroups became more accessible to the lipid headgroups. In order to have a more clear picture about the exclusion zones, and the preferential positioning of the lipid particles in the vicinity of the gemini molecules, we resorted to the representation of density maps and repulsion energy contour profiles (Figure 6). The average position of the lipids was obtained from separate simulations for one fixed surfactant (i.e. the surfactant molecule was not allowed to move throughout the simulation) with a 

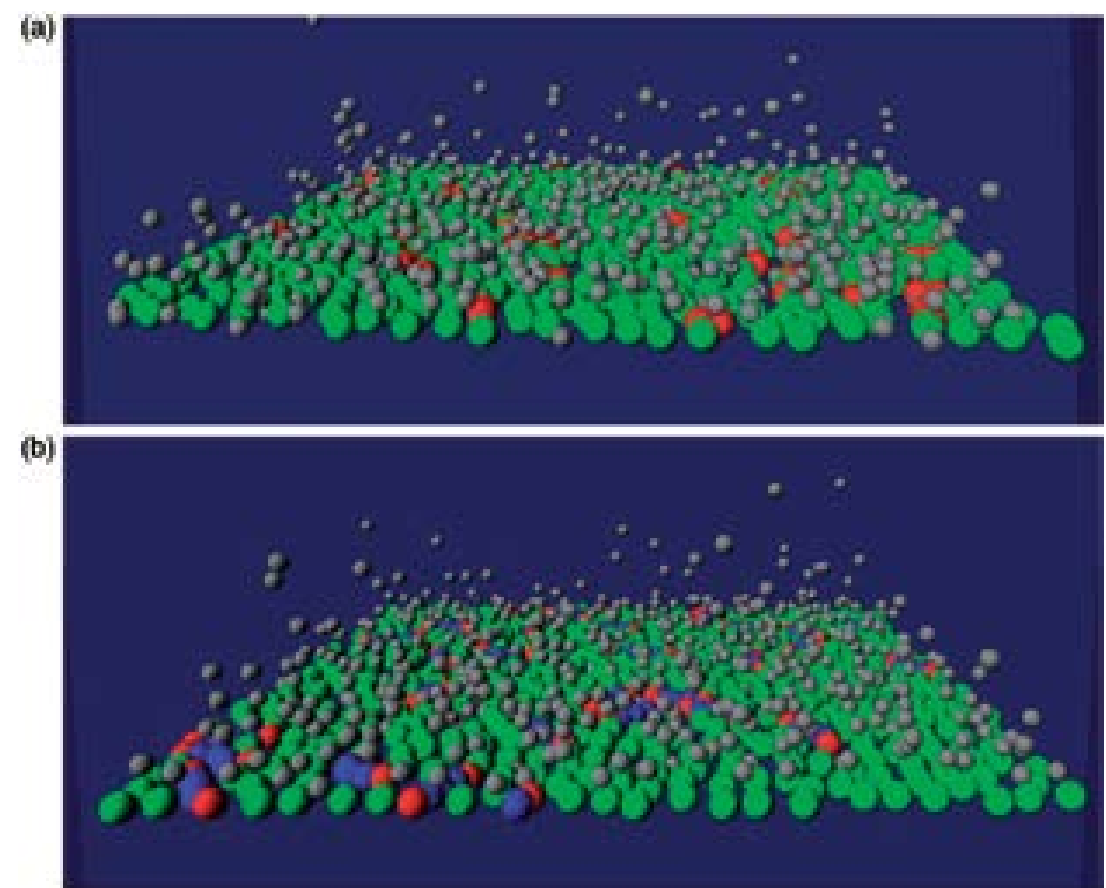

(c)

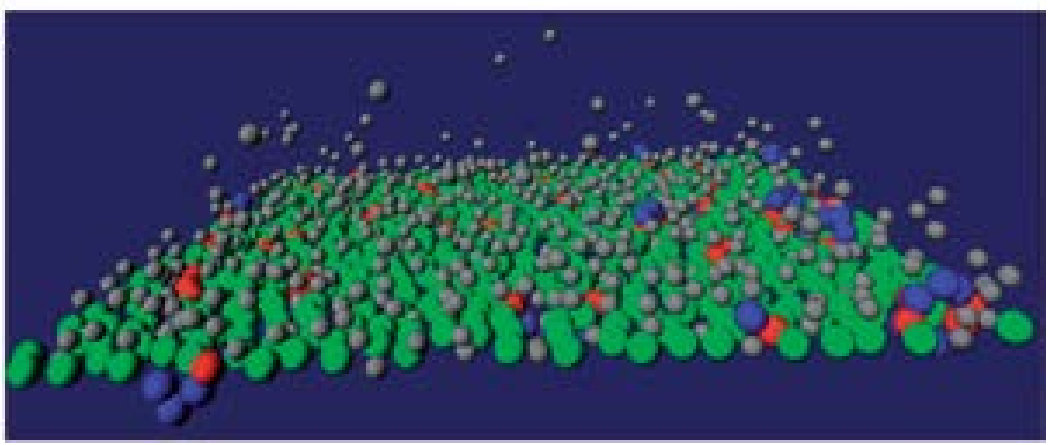

Figure 5. Representative snapshots of a positively charged membrane, green, containing $10.8 \%$ of gemini surfactants, red (charged) and blue (neutral), and respective counterions, grey. Both lipid and gemini particles possess a hs radius of $3.3 \AA$ and the counterions have a hs radius of $2.2 \AA$. (a) Surfactant with the shortest spacer (II); (b) surfactant with the longest spacer restricted to the $x y$ plane (VI); (c) surfactant with the longest spacer not restricted to the $x y$ plane (VII).

representative conformation and the contours of the coulombic energy were calculated based on the average end-to-end distance of each system. The maps clearly show the excluded zones and the concentric shells. The excluded zones correspond to the higher hs repulsion regions, while the concentric shells evidence the areas with higher probability to find the lipid headgroups. Note the similarity between the concentric shells and the energy contours obtained from the coulombic energy repulsion, calculated without taking into account the volume of the hard sphere. In the shorter spacer surfactant the two headgroups are substantially overlapped giving rise to almost circular energy contours, which are similar to the respective concentric shells in the left panels. Regarding the surfactants with longer spacer, the overall excluded volume areas are higher. However, the presence of the neutral spacer intermediating the two charged headgroups promotes a decrease of the repulsion between the two headgroups and, consequently, a larger number of lipid particles are allowed in their vicinity. This can be clearly seen in the regions represented in yellow in the positioning maps and in the panels corresponding to the coulombic energies. The repulsion energy values for the gemini-lipid (G-L) interaction (see Table 3) follow the behaviour depicted in the rdfs, the repulsion energy increases as the components became closer to each other.

Let us now look at the distribution of the counterions around the lipid and surfactant headgroups 

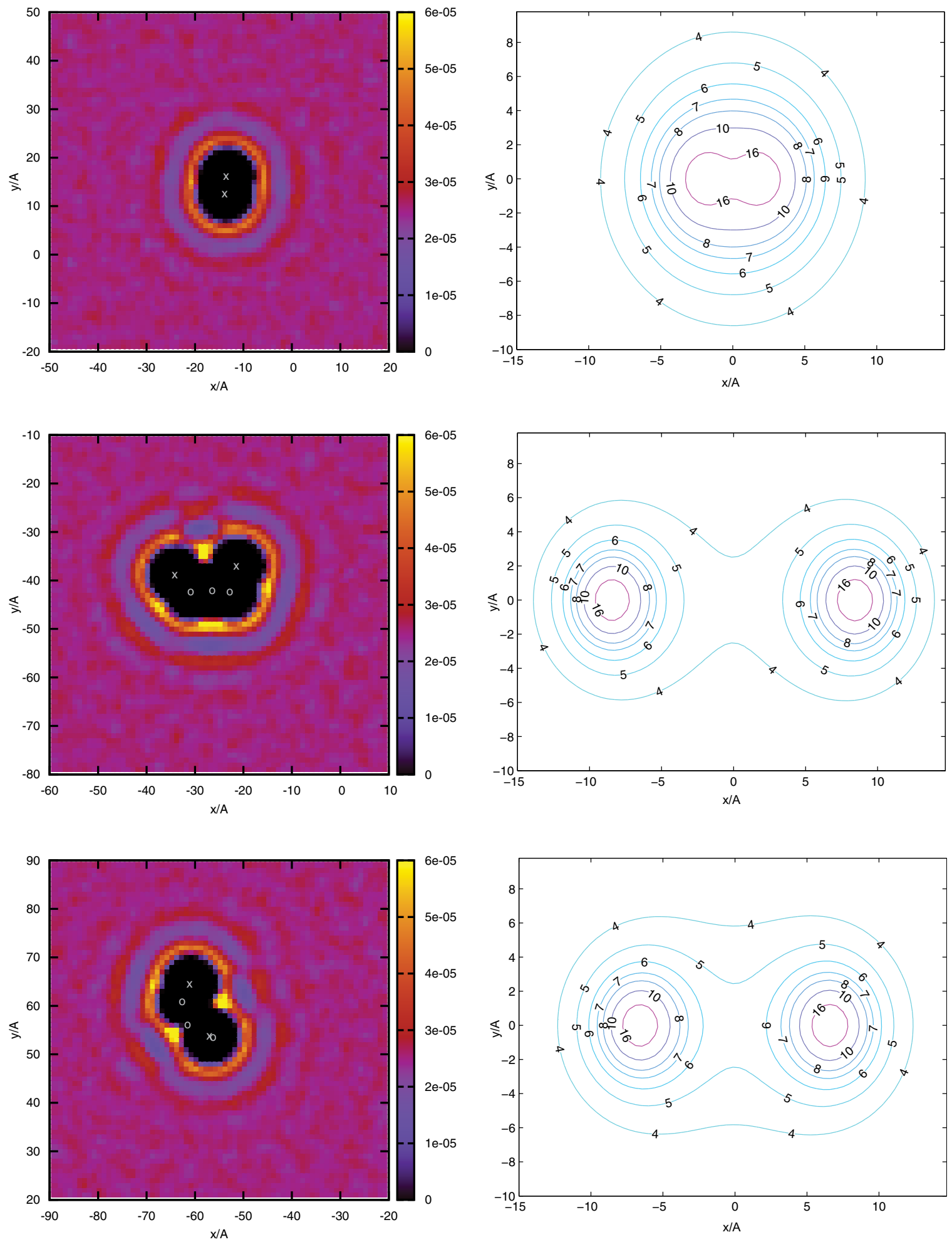

Figure 6. Left panels: Positioning maps of the lipid headgroups around the gemini surfactant for systems, from top to bottom, shortest spacer, longer spacer restricted to the membrane plane, and longer non-restricted spacer. ' $\mathrm{x}$ ' denotes the position of the gemini headgroup and 'o' denotes the neutral groups corresponding to the spacer, or its projection on the $x y$ plane for nonrestricted spacer. Right panels: Energy contours of the coulombic repulsion energy $\left(\mathrm{kJ} \mathrm{mol}^{-1}\right)$ calculated according to the $\left\langle R_{\mathrm{ee}}^{2}\right\rangle^{1 / 2}$ value of each system. 


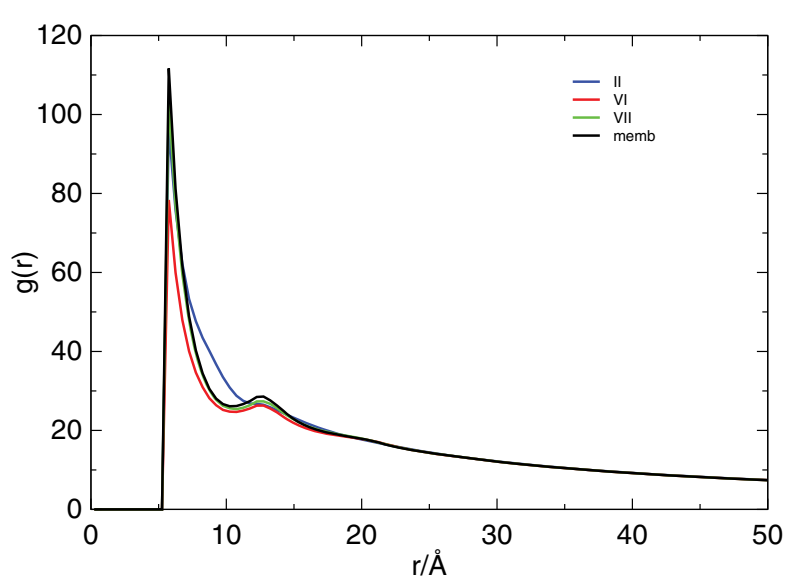

Figure 7. 3D-rdf of the counterions relative to the gemini headgroups, for systems containing $10.8 \%$ of gemini with different spacer lengths. The 3D-rdf curve of the counterions relative to the lipid headgroups was also included (memb).

(3D-rdf analyses, Figure 7). The profile of the distribution of the counterions around lipid headgroups, presented in the black curve (memb), does not vary with the incorporation of gemini surfactants, independently of their concentration and spacer length, at least within the studied systems (data not shown). The same profile is also observed for the distribution of the counterions around the gemini headgroups for systems with the longest spacer (VI, VII). However, in these cases there is a decrease in the number of condensed counterions, which can be related with the fact that, in system VI, the presence of the neutral spacer in the membrane plane tends to decrease the charge density around the gemini particles, thus reducing the condensation of counterions. When the spacer is allowed to move along the $z$-axis, system VII, a slight increase of the counterion condensation is observed. This may be attributed to the decrease in the hs density at the membrane level, which allows a greater penetration of counterions, combined with a potential increase in the charge density of the gemini headgroups. The latter is justified by the larger conformational freedom of the spacer (see Figure 4). Finally, system II shows a slightly different profile, indicating a larger condensation of counterions to the gemini surfactants with the shorter spacers. This is in good agreement with the results shown in Table 3 and also with the results reported in [32], where it was noted a preference for the counterions to be near the headgroups of the gemini with the smallest spacer. This preference has been attributed to the higher charge density and to the protruded position of these groups relative to the average level of the membrane surface [32]. Although

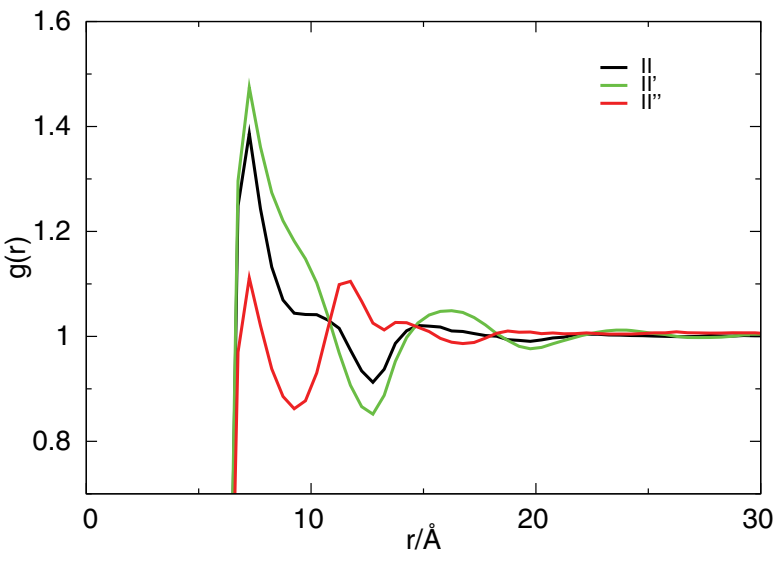

Figure 8. 2D-rdf accounting for the distribution of the lipid headgroups relative to the gemini headgroups in systems containing $10.8 \%$ of gemini surfactants (black line: gemini and lipid headgroups with charge +1 ; green line: neutral gemini and lipid headgroups; red line: gemini headgroups with charge +2 and lipid headgroups with charge +1$)$.

the latter effect could not be accounted for in this model, the trend could still be reproduced. These results were also confirmed resorting to nearestneighbour distribution function analysis (data not shown), with the number of counterions condensed around the gemini headgroups following the order II $>$ VII $>$ VI.

\subsection{Electrostatic vs excluded volume effects}

Let us now return to the gemini-lipid rdf depicted in Figure 2, panel (c). It was noted that in system II the main peak has a pronounced shoulder that extends to larger distances. MD studies of similar systems have shown the same type of profile, and it was suggested that the shoulder may result from the increased repulsion between gemini and lipid headgroups, due to the higher charge density present in the shorter spacer gemini molecules [32]. To clarify this point, we have resorted to the flexibility of the coarse-grained model. Therefore, two other systems with short spacer gemini were calculated based on system II but with varying charge of the gemini and/or of the lipid headgroups. In one of the systems, all particles are neutral (system $\mathrm{II}^{\prime}$ ) and in the other the gemini headgroups have a double positive charge while the lipid headgroups keep the unitary charge (system II"). With these variations, we hope to evaluate the relative importance of electrostatic and excluded volume effects on such systems. The 2D-rdfs accounting for the gemini-lipid interactions are represented in 

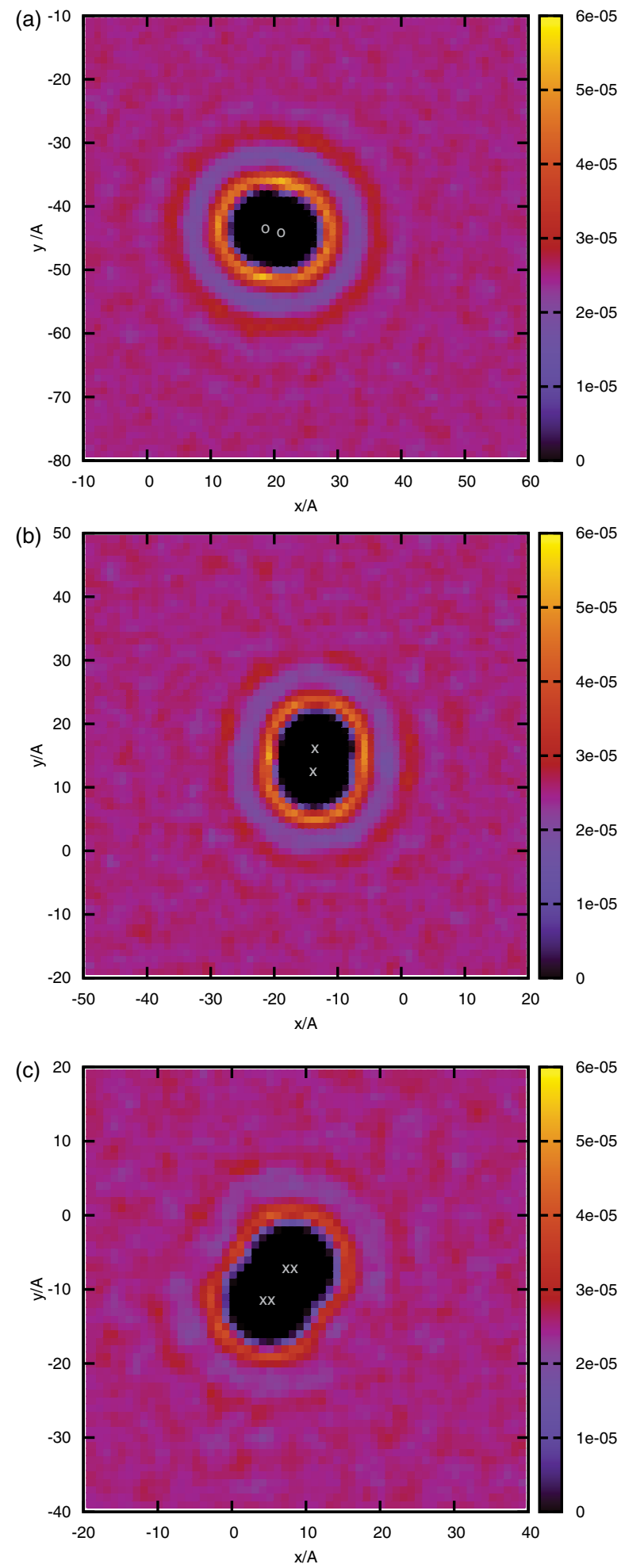

Figure 9. Density maps showing the probability of the arrangement of the lipid headgroups around one gemini surfactant (frozen at a representative conformation). ' $\mathrm{o}$ ', ' $\mathrm{x}$ ' and ' $x x^{\prime}$ ' denotes, in each case, the position of the gemini headgroups. (a) Neutral gemini and lipid headgroups; (b) gemini and lipid headgroups with unitary charge; (c) gemini and lipid headgroups with charge +2 and +1 , respectively.
Figure 8, where the data for system II is also included. It can be seen that although the maxima of the first peak are located at approximately the same value, $c a$. $7.3 \AA$ for the three systems, marked differences are found between them. The decrease in the peak height with the increase of the gemini headgroup charge can be attributed to the increased repulsion with the lipid headgroups, that tends to lower the density of lipid molecules in the close vicinity of the surfactant headgroups. Furthermore, the shoulder coupled to the first peak, that has been identified in system II, is still present when all the species in the system are neutral (system II'), and becomes a well-defined peak when the charge of the gemini headgroup is increased from +1 to +2 (system $\mathrm{II}^{\prime \prime}$ ). It becomes evident that, besides the electrostatic effect that tends to repel the lipid headgroups to longer distances when the charge of the gemini headgroup increases, other effects play a role in the organization of the system. The organiza-

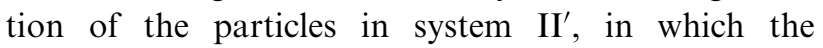
electrostatic effects are absent, clearly indicates the role of excluded volume.

In Figure 9, the arrangement of the lipid headgroups (with zero or unitary charge) around one gemini molecule with different headgroup charges is evidenced. As expected, the increase of the charge on the gemini headgroups, promotes a larger excluded volume. In comparison with the original system, with unitary charges, (Figure 9, panel (b)), the fully neutral system (Figure 9, panel (a)) shows that the absence of electrostatic effects promotes a larger concentration of lipids around the surfactant headgroups as well as a more long-ranged organization. Note the larger amplitude of the second peak in the rdf (Figure 8). On the other hand, when the electrostatic effects were intensified (Figure 9, panel (c)), the system appears to be less ordered.

The interpretation of the rdf profiles can be made resorting to a simple schematic representation, presented in Figure 10, where the data corresponding to the $\left\langle R_{\mathrm{ee}}^{2}\right\rangle^{1 / 2}$ of each system are also included. The first peak, which is common to the three systems, corresponds to the distance between one gemini headgroup and the neighbouring lipid headgroup (blue dashed and dotted lines in Figure 10), while the position of the shoulder or second peak, that varies from one system to another, corresponds to the distance from the other gemini headgroup of the same gemini molecule to the same layer of surrounding lipid molecules (white dashed lines in Figure 10). Note that the variety of distances in the latter case (represented by white dashed lines) is responsible for the broadening of the shoulder/second peak. 

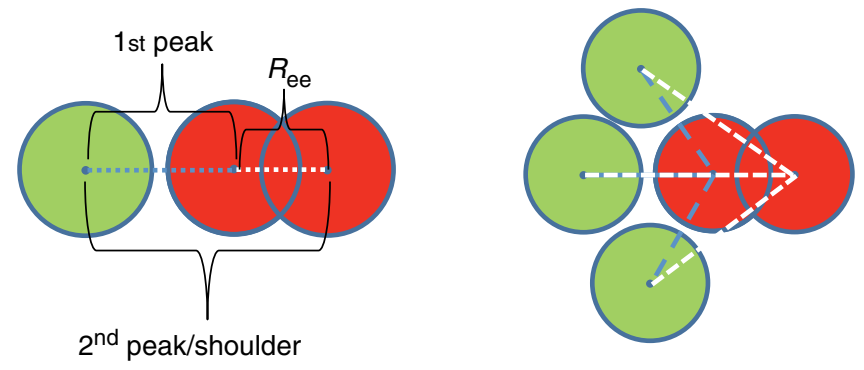

\begin{tabular}{lccc}
\hline & Gemini headgroup charge & Lipid headgroup charge & $R_{\mathrm{ee}} / \AA$ \\
\hline II' & 0 & 0 & 3.504 \\
II & +1 & +1 & 3.775 \\
II" & +2 & +1 & 4.507 \\
\hline
\end{tabular}

Figure 10. Schematic representation of the distribution of the lipid headgroups (green circles) around a gemini molecule (red circles) with different headgroup charges. Values of the end-to-end distance, $\left\langle R_{\mathrm{ee}}^{2}\right\rangle^{1 / 2}$, for each case, are also included.

The presence of the shoulder is thus attributed to excluded volume effects, due to the overlapping of the surfactant headgroups, while its width and position are mainly governed by electrostatic effects.

\section{Conclusions}

A selected set of systems comprising different cationic gemini surfactants incorporated in a positively charged lipid-like membrane were simulated resorting to a simple model using a spring-bead chain to represent the surfactants, and a surface carrying positive charges which mimicked the surface of a charged membrane. The coarse-grained approach was used with the purpose of studying the influence of gemini concentration and spacer length on the distribution and organization of the lipid particles. The present work shows that, at the membrane surface level, the surfactant concentration and the spacer length have a small influence in the organization of the lipid headgroups relative to each other, which reflects the adaptability of the systems in this model. On the other hand, the organization of the lipid headgroups in the vicinity of a gemini surfactant strongly depends on the surfactant spacer length. Surfactants with longer spacers that are restricted to the membrane plane, promote an increase in the order of the lipid groups due to an increase in the hardsphere density. Furthermore, the decrease of the charge density near the gemini headgroups upon the increase in the surfactant spacer length favours the approximation of the lipid headgroups to the surfactant and diminishes the adsorption of counterions. When the spacer is allowed to move along the $z$-axis, the lipid headgroups are, on average, even closer to the surfactant in comparison to the restricted surfactant, and the adsorption of counterions is now also favoured. Taking advantage of the simplicity of the used model, the factors responsible for the profiles observed in the rdfs could be discriminated. The gemini surfactant intramolecular and intermolecular contributions could be discriminated on the profiles of the geminigemini rdfs. Additionally, variations in the charge of the gemini surfactants and lipid headgroups, in the shortest spacer surfactant, allowed one to elucidate the role of the electrostatic and excluded volume effects in the organization of the lipid headgroups around the surfactant. The results suggest that the distribution of the particles in these systems is governed not only by the electrostatic effects, but by an interplay between electrostatic contribution and excluded volume effects.

\section{Acknowledgements}

This work was supported by FEDER funds through the COMPETE program - Programa Operacional Factores de Competitividade - and by National funds through Fundaça para a Ciencia e Tecnologia (FCT) under the Project PTDC/ QUI-QUI/101442/2008 (COMPETE:FCOMP-01-0124FEDER-010831). S.C.C.N. and J.A.S.A. gratefully acknowledge the post-doctoral research grants SFRH/BPD/71683/ 2010 and SFRH/BPD/82125/2011, respectively, and R.S.D. acknowledges the program Ciência 2007, all assigned by the Fundação para a Ciência e Tecnologia (FCT). 


\section{References}

[1] L. Wasungu, M. Scarzello, G. van Dam, G. Molema, A. Wagenaar, J.B.F.N. Engberts and D. Hoekstra, J. Mol. Med. 84, 774 (2006).

[2] A.J. Kirby, P. Camilleri, J.B.F.N. Engberts, M.C. Feiters, R.J.M. Nolte, O. Söderman, M. Bergsma, P.C. Bell, M.L. Fielden, C.L. García Rodríguez, P. Guédat, A. Kremer, C. McGregor, C. Perrin, G. Ronsin and M.C.P. van Eijk, Angew. Chem. Int. Ed. 42, 1448 (2003).

[3] B.K. Kim, K.O. Doh, Y.U. Bae and Y.B. Seu, J. Microbiol. Biotechnol. 21, 93 (2011).

[4] P. Yang, J. Singh, S. Wettig, M. Foldvari, R.E. Verrall and I. Badea, Eur. J. Pharm. Biopharm. 75, 311 (2010).

[5] A.M.S. Cardoso, H. Faneca, J.A.S. Almeida, A.A.C.C. Pais, E.F. Marques, M.C.P. Lima and A.S. Jurado, Biochim. Biophys. Acta 1808, 341 (2011).

[6] S.D. Wettig, R.E. Verrall and M. Foldvari, Curr. Gene Ther. 8, 9 (2008).

[7] C. Bombelli, L. Giansanti, P. Luciani and G. Mancini, Curr. Med. Chem. 16, 171 (2009).

[8] A. Colomer, A. Pinazo, M.A. Manresa, M.P. Vinardell, M. Mitjans, M.R. Infante and L. Pérez, J. Med. Chem. 54, 989 (2011).

[9] N.A. Negm, M.F. Zaki and M.A.I. Salem, J. Dispersion Sci. Technol. 31, 1390 (2010).

[10] M.A. Hegazy, M. Abdallah and H. Ahmed, Corros. Sci. 52, 2897 (2010).

[11] F.A. Ansari and M.A. Quraishi, Arab. J. Sci. Eng. 36, 11 (2011).

[12] Z. Huang, H. Lu, T. Zhang, R. Wang and D. Qing, Pet. Sci. Technol. 28, 1621 (2010).

[13] M.N. Maithufi, D.J. Joubert and B. Klumperman, Energy Fuels 25, 162 (2011).

[14] B. Weng, R. Shepherd, J. Chen and G.G. Wallace, J. Mater. Chem. 21, 1918 (2011).

[15] M. In and R. Zana, J. Dispersion Sci. Technol. 28, 143 (2007).

[16] M. Johnsson and J.B.F.N. Engberts, J. Phys. Org. Chem. 17, 934 (2004).

[17] S.K. Hait and S.P. Moulik, Curr. Sci. 82, 1101 (2002).

[18] F.M. Menger and J.S. Keiper, Angew. Chem. Int. Ed. 39, 1906 (2000).
[19] S. Zhu, L. Liu and F. Cheng, J. Surfactants Deterg. 14, 221 (2011).

[20] X.P. Liu, J. Feng, L. Zhang, Q.T. Gong, S. Zhao and J.Y. Yu, Colloids Surf., A 362, 39 (2010).

[21] M. Cai, M. Zhang and P. Ma, J. Dispersion Sci. Technol. 31, 1633 (2010).

[22] C.F.J. Kuo, L.H. Lin, M.Y. Dong, W.S. Chang and K.M. Chen, J. Surfactants Deterg. 14, 195 (2011).

[23] T.R. Prytkova, I. Eryazici, B. Stepp, S. Nguyen and G.C. Schatz, J. Phys. Chem. B 114, 2627 (2010).

[24] J.H. Allen, E.T. Schoch and J.M. Stubbs, J. Phys. Chem. B 115, 1720 (2011).

[25] M. Muller, K. Katsov and M. Schick, Phys. Rep. 434, 113 (2006).

[26] S.V. Bennun, M.I. Hoppes, C. Xing and R. Faller, Chem. Phys. Lipids 159, 59 (2009).

[27] K. Patterson, M. Lisal and C.M. Colina, Fluid Phase Equilib. 61, 48 (2011).

[28] J. Zhang, W. Li, J. Wang, M. Qin, L. Wu, Z. Yan, W. Xu, G. Zuo and W. Wang, IUBMB Life 61, 627 (2009).

[29] G. Bellesia, A.I. Jewett and J.E. Shea, Protein Sci. 20, 818 (2011).

[30] F. Schmid, Macromol. Rapid Commun. 30, 741 (2009).

[31] M.J. Stevens, J. Chem. Phys. 121, 11942 (2004).

[32] J.A.S. Almeida, S.R. Pinto, Y. Wang, E.F. Marques and A.A.C.C. Pais, Phys. Chem. Chem. Phys. 13, 13772 (2011).

[33] J.A.S. Almeida, H. Faneca, R.A. Carvalho, E.F. Marques and A.A.C.C. Pais, PLoS ONE 6 (1), e26965 (2011).

[34] J.A.S. Almeida, E.F. Marques, A.S. Jurado and A.A.C.C. Pais, Phys. Chem. Chem. Phys. 12, 14462 (2010).

[35] Y. Marcus, J. Chem. Soc. Faraday Trans. 87, 2995 (1991).

[36] P. Pullmannová, M. Bastos, G. Bai, S.S. Funari, I. Lacko, F. Devínsky, J. Teixeira and D. Uhríková, Biophys. Chem. 160, 35 (2012).

[37] R. Ionov, A. El-Abed and M. Goldmann, Eur. Biophys. J. 38, 229 (2009).

[38] P. Linse, MOLSIM, version 4.0.8 (2004). Available from: http://130.235.71.204/home.php?Program=Molsim.

[39] M.P. Allen and D.J. Tildesley, Computer Simulation of Liquids (Clarendon, Oxford, 1987). 